\title{
A Pseudoinverse Update Algorithm for Rank-Reduced Covariance Matrices from 2-D Data
}

\author{
Gregory Robertson, Member, IEEE, R. Lynn Kirlin, Senior Member, IEEE, and W.-S. Lu, Senior Member, IEEE
}

\begin{abstract}
A number of algorithms have a higher resolution than the common beamformer. These often require the calculation of a pseudoinverse of a matrix, which makes the algorithm very slow for repeated applications. We consider updating the pseudoinverse for window motions either in time or in space for two-dimensional (2-D) data taken from a linear array. Our results are shown to reduce the computational complexity of the multiple sidelobe canceller (MSC) [1], for example, by more than $\mathbf{7 5 \%}$ for a downward window movement (with time) and more than $55 \%$ for a sideways window movement (across traces).
\end{abstract}

Index Terms - Pseudoinverse update, covariance matrix.

\section{INTRODUCTION}

A POTENTIAL problem with several high-resolution algorithms is the large number of calculations necessary for determining the pseudoinverse of the auxiliary correlation matrix when a square blocking matrix is used. We provide economical methods for not only the down-trace or timeupdate analysis, but also the much more complicated crosstrace or spatial update analysis. Our work adds to that for one-dimensional (1-D) data updates [3]. We will apply the matrix inversion lemma [2]

$$
\begin{aligned}
M^{-1} & =(A+B D C)^{-1} \\
& =A^{-1}-A^{-1} B\left(D^{-1}+C A^{-1} B\right)^{-1} C A^{-1} .
\end{aligned}
$$

Consider the sliding window to be a frame that encloses the data matrix $X, M$ sensors (traces) and $N$ time samples

$$
X=\left[\bar{x}_{1} \bar{x}_{2} \cdots \bar{x}_{N}\right]=\left[\begin{array}{cccc}
x_{1,1} & x_{1,2} & \cdots & x_{1, N} \\
x_{2,1} & x_{2,2} & \cdots & x_{2, N} \\
\vdots & \vdots & \vdots & \vdots \\
x_{M, 1} & x_{M, 2} & \cdots & x_{M, N}
\end{array}\right]
$$

having sample correlation matrix $R_{x}=X X^{T} / N$. The sample correlation matrix at position $k-1$ relates to the sample correlation matrix at $k$ with the use of a difference matrix, i.e.,

$$
R_{x}^{(k)}=R_{x}^{(k-1)}+R_{\triangle}^{(k)} .
$$

We will identify parts of (3) with (1) for the inversion.

Manuscript received November 13, 1996. The associate editor coordinating the review of this manuscript and approving it for publication was Prof. K. Buckley.

The authors are with the Department of Electrical and Computer Engineering, University of Victoria, Victoria, B.C., Canada V8W 3P6 (e-mail: kirlin@ece.uvic.ca).

Publisher Item Identifier S 1070-9908(97)05976-2.

\section{Down-Trace or Time Update Analysis}

The easier of the two analysis methods is the analysis downtrace (or with time) due to the fact that one entire column vector is dropped and one entire vector is added during each move of the window. When one defines the dropped vector $x_{\text {old }}$ as the first column vector from the previous window matrix $\mathrm{X}^{k-1}$ and the added vector $x_{n e w}$ as the last column vector from the current window matrix $\mathbf{X}^{(k)}$, then $R_{\triangle}$ in (3) becomes

$$
\begin{aligned}
R_{\triangle}^{(k)} & =\left(x_{n e w} x_{\text {new }}^{T}-x_{o l d} x_{o l d}^{T}\right) \\
& =U^{(k)} \cdot \hat{I}_{2} \cdot U^{(k)^{T}}
\end{aligned}
$$

where $\hat{I}_{2}=\operatorname{diag}\{1,-1\}$, and $U^{(k)}=\left[x_{\text {new }} x_{\text {old }}\right]$.

Multiplying $U^{(k)}$ by the blocking matrix $B=I_{M}-u$. $u^{T} / M$, where $u$ is a vector of ones, gives auxiliary matrix

$$
U_{a}^{(k)}=B \cdot U^{(k)} \text {. }
$$

(We have predelayed or beam-steered the data to the trial signal source directions thus the unity direction vector $u$.) With these definitions, the current auxiliary correlation matrix can be written as

$$
R_{a}^{(k)}=R_{a}^{(k-1)}+U_{a}^{(k)} \cdot \hat{I}_{2} \cdot U_{a}^{(k)^{T}} .
$$

Applying (1) to (6) gives the desired pseudoinverse update

$$
R_{a}^{\dagger(k)}=R_{a}^{\dagger(k-1)} \cdot\left(I_{M}-C\right)
$$

where $C=U_{a}^{(k)} \cdot A^{-1} \cdot U_{a}^{(k)^{T}} \cdot R_{a}^{\dagger(k-1)}$, and $A=\hat{I}_{2}+U_{a}^{(k)^{T}}$. $R_{a}^{\dagger(k-1)} \cdot U_{a}^{(k)}$.

\section{Cross-Trace or Spatial Update Analysis}

Cross-trace window motion is appropriate when analyzing spatial variations in wavefronts such as with seismic data. For cross-trace analysis the problem has changed so that upon each new iteration each column vector in the window gains one new sensor element and loses one old sensor element, causing diagonally upward shift in the values of the correlation matrix. Defining the $M \times M$ shifting matrix

$$
S=\left[\begin{array}{cccccccc}
0 & 1 & 0 & 0 & 0 & \cdots & 0 & 0 \\
0 & 0 & 1 & 0 & 0 & \cdots & 0 & 0 \\
\vdots & \vdots & \vdots & \vdots & \vdots & \cdots & \vdots & \vdots \\
0 & 0 & 0 & 0 & 0 & \cdots & 0 & 1 \\
1 & 0 & 0 & 0 & 0 & \cdots & 0 & 0
\end{array}\right]
$$

the interim shifted version of the pseudoinverse of $R_{a}$ is

$$
\vec{R}_{a}^{\dagger(k-1)}=S \cdot R_{a}^{\dagger(k-1)} \cdot S^{T} .
$$


If one applies the shifting matrix to the $R_{x}^{(k-1)}$ in (3) the difference matrix becomes

$$
R_{\triangle}^{(k)}=\left[\begin{array}{ccccc}
0 & 0 & \cdots & 0 & a_{1} \\
0 & 0 & \cdots & 0 & a_{2} \\
\vdots & \vdots & \cdots & \vdots & \vdots \\
0 & 0 & \cdots & 0 & a_{M-1} \\
a_{1} & a_{2} & \cdots & a_{M-1} & a_{M}
\end{array}\right]
$$

where each element, $\left[a_{1}, a_{2}, \cdots, a_{M}\right]$ is the difference between two scalars from within the two sequential correlation matrices.

Further, the window matrix $X$ drops row $y_{\text {old }}=$ $\left[X_{(1,\{1,2, \cdots, N\})}^{(k-1)}\right]^{T}$, and adds row $y_{\text {new }}=$ $\left[X_{(M,\{1,2, \cdots, N\})}^{(k)}\right]^{T}$. Now defining

$$
\begin{aligned}
\delta & =\frac{\left(y_{\text {new }}-y_{\text {old }}\right)}{N} \\
c_{1} & =\sqrt{\frac{\left(y_{\text {new }}^{T} \cdot y_{\text {new }}\right)}{N}} \\
c_{2} & =\sqrt{\frac{\left(y_{\text {old }}^{T} \cdot y_{\text {old }}\right)}{N}} \\
c_{3} & =c_{1}+c_{2} .
\end{aligned}
$$

gives similarly as in (4)-(6)

$$
R_{a}^{(k)}=S \cdot R_{a}^{(k-1)} \cdot S^{T}+U_{a}^{(k)} \cdot \hat{I}_{2} \cdot U_{a}^{(k)^{T}}
$$

where

$$
\begin{aligned}
U^{(k)} & =[\alpha \beta], \\
\alpha & =\frac{X^{(k)} \cdot \delta}{c_{3}} \\
\beta_{\{1,2, \cdots, M-1\}} & =\alpha_{\{1,2, \ldots, M-1\}} \\
\alpha_{M} & =c_{1} \\
\beta_{M} & =-c_{2} .
\end{aligned}
$$

Applying (1) gives the cross-trace pseudoinverse update

$$
\vec{R}_{a}^{\dagger(k)}=\vec{R}_{a}^{\dagger(k-1)} \cdot\left(I_{M}-C\right)
$$

where $C=U_{a}^{(k)} \cdot A^{-1} U_{a}^{(k)^{T}} \cdot \vec{R}_{a}^{\dagger(k-1)}$, and $A=\hat{I}_{2}+U_{a}^{(k)^{T}}$. $\vec{R}_{a}^{\dagger(k-1)} \cdot U_{a}^{(k)}$.

Although numerical instability might occur when matrixinversion-lemma-based update is employed [5], we note that the matrix inversion in both (7) and (14) only involves wellconditioned symmetric matrices of size $2 \times 2$, leading to numerical robustness as well as substantially reduced computational complexity.

\section{RESULTS AND CONCLUSIONS}

In Figs. 1 and 2, results are presented as a percentage of the flop count ratios of i) the beamformer (semblance) method and ii) the updating multiple sidelobe canceller (MSC) method to the conventional MSC method. As one can observe in both

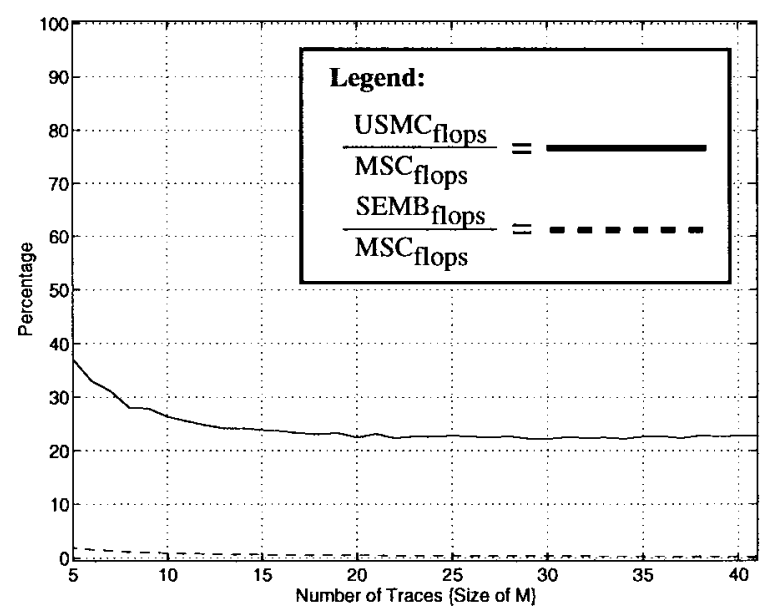

Fig. 1. Flop count ratio for time update analysis.

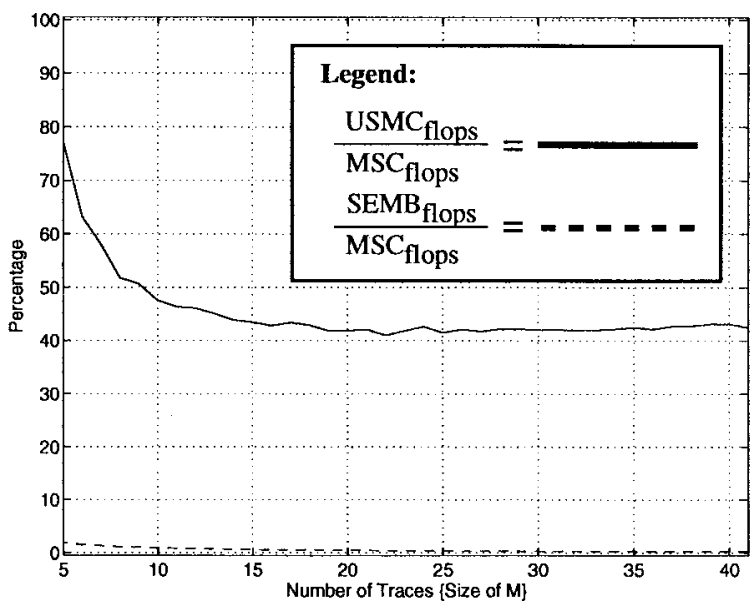

Fig. 2. Flop count ratio for spatial update analysis.

figures, the value of $M$ increases with a fixed number of time samples $(N=49)$, the efficiency of the updating MSC increases as compared to the MSC algorithm. In Fig. 1, with a value of $M$ at 25 or more, the average number of flops for the down-trace updating MSC is only about $22.56 \%$ of that of the MSC algorithm. For the cross-trace analysis results and the same $M$ values, Fig. 2 shows the average number of updating MSC flops to be about $42.60 \%$ of that of the MSC algorithm.

\section{REFERENCES}

[1] B. D. Van Veen and K. M. Buckley, "Beamforming: A versatile approach to spatial filtering," IEEE Acoust., Speech, Signal Processing Mag., vol. 5, pp. 4-24, Apr. 1988.

[2] S. Haykin, Adaptive Filter Theory. Englewood Cliffs, NJ: PrenticeHall, 1991.

[3] M. Mahon, L. Sibul, and H. Valenzuela, "A sliding window update for the basis matrix of the QR decomposition," IEEE Trans. Signal Processing, vol. 41, pp. 1951-1953, May 1993.

[4] R. L. Kirlin, "Preliminary considerations on high resolution moving window multiple coherency estimators," Rep., Amoco Inc., Mar. 1993.

[5] K. M. Buckley, private communication, Apr. 1997. 